OPEN ACCESS

Edited by:

Payal Mukherjee,

The University of Sydney, Australia

Reviewed by:

Antonello Forgione,

Independent Researcher, Milan, Italy Andrew Gumbs,

Département de Chirurgie Viscérale, France

*Correspondence:

Jasamine Coles-Black JasamineCB@gmail.com

Specialty section:

This article was submitted to Visceral Surgery

a section of the journal

Frontiers in Surgery

Received: 05 November 2020 Accepted: 07 December 2020 Published: 27 January 2021

Citation:

Coles-Black J, Bolton D and Chuen J (2021) Accessing 3D Printed Vascular Phantoms for Procedural Simulation.

Front. Surg. 7:626212.

doi: 10.3389/fsurg.2020.626212

\section{Accessing 3D Printed Vascular Phantoms for Procedural Simulation}

\author{
Jasamine Coles-Black ${ }^{1,2 *}$, Damien Bolton ${ }^{2}$ and Jason Chuen ${ }^{1,2}$ \\ ${ }^{1} 3 d$ MedLab, Austin Health, The University of Melbourne, Parkville, VIC, Australia, ${ }^{2}$ Department of Surgery, Austin Health, The \\ University of Melbourne, Melbourne, VIC, Australia
}

Introduction: 3D printed patient-specific vascular phantoms provide superior anatomical insights for simulating complex endovascular procedures. Currently, lack of exposure to the technology poses a barrier for adoption. We offer an accessible, lowcost guide to producing vascular anatomical models using routine CT angiography, open source software packages and a variety of 3D printing technologies.

Methods: Although applicable to all vascular territories, we illustrate our methodology using Abdominal Aortic Aneurysms (AAAs) due to the strong interest in this area. CT aortograms acquired as part of routine care were converted to representative patientspecific 3D models, and then printed using a variety of 3D printing technologies to assess their material suitability as aortic phantoms. Depending on the technology, phantoms cost $\$ 20-\$ 1,000$ and were produced in 12-48 h. This technique was used to generate hollow 3D printed thoracoabdominal aortas visible under fluoroscopy.

Results: 3D printed AAA phantoms were a valuable addition to standard CT angiogram reconstructions in the simulation of complex cases, such as short or very angulated necks, or for positioning fenestrations in juxtarenal aneurysms. Hollow flexible models were particularly useful for device selection and in planning of fenestrated EVAR. In addition, these models have demonstrated utility other settings, such as patient education and engagement, and trainee and anatomical education. Further study is required to establish a material with optimal cost, haptic and fluoroscopic fidelity.

Conclusion: We share our experiences and methodology for developing inexpensive 3D printed vascular phantoms which despite material limitations, successfully mimic the procedural challenges encountered during live endovascular surgery. As the technology continues to improve, 3D printed vascular phantoms have the potential to disrupt how endovascular procedures are planned and taught.

Keywords: 3D printing, vascular phantom, simulation, fluoroscopy, angiography, AAA (abdominal aortic aneurysm), EVAR, FEVAR

\section{INTRODUCTION}

\section{D Printed Vascular Phantoms}

$3 \mathrm{D}$ printing is a manufacturing technique which has gained attention in surgery recently as a means of rapidly producing patient-specific anatomical models for the purposes of procedural simulation and training. This accessible technology allows imaging to be converted into physical, patient-specific models within the hospital setting, enabling surgeons and other proceduralists 
to rapidly access true-to-scale representations of patient anatomy for superior visualization and planning.

$3 \mathrm{D}$ printing is anticipated to represent the next step in personalized medicine. Despite the nascence of the technology, its utility as a tool in presurgical planning and intraoperative visualization is currently being examined via clinical trials (1). In addition, its potential in other settings is being explored, such as for patient education and engagement and trainee education. Due to the expiry of patents leading to the democratization of $3 \mathrm{D}$ printing technology, desktop $3 \mathrm{D}$ printers are now in the price range of office paper printers, well within reach of all surgical departments seeking to produce of patient-specific 3D printed anatomical models in-house. In the field of vascular intervention, $3 \mathrm{D}$ printed models have been used as presurgical simulation tools in the planning of Endovascular Aneurysm Repair (EVAR) (2), and complex endovascular aortic techniques such as fenestrated or branched $\operatorname{EVAR}(3,4)$. 3D printed vascular models have also been explored in procedural simulation involving other vascular territories, such as coil embolisation of cerebral aneurysms (5) or splenic artery aneurysms (6).

Our frontier experiences with the technology mirror those of other groups, with 3D printed patient-specific vascular models providing superior anatomical insights for simulating complex procedures (Figure 1). This has clear advantages regarding patient safety with reduced time under anesthesia, reduction in operation time (7), shorter recovery times, and a reduction in blood loss intraoperatively (8), resulting in cost savings to health services. However, despite the promise of $3 \mathrm{D}$ printed patient-specific phantoms for simulation, lack of exposure to the technology amongst vascular proceduralists poses a barrier for adoption (9).

We offer an accessible, low-cost guide to producing vascular anatomical models using routine CT angiography, open source software packages and a variety of $3 \mathrm{D}$ printing technologies, with a focus on Abdominal Aortic Aneurysms (AAAs) to showcase its utility. However, the techniques described are equally applicable to any vascular territory imaged using Computed Tomographic (CT) angiography.

\section{Abdominal Aortic Aneurysms}

An Abdominal Aortic Aneurysm is a dilatation of the abdominal aorta to at least 1.5 times its usual size, or an outer diameter of $3 \mathrm{~cm}$ (10). As a true aneurysm, the dilatation affects all three layers of the aortic wall, namely the intima, media, and adventitia. AAAs cause a substantial burden on the healthcare system. Rupture results in death in $65 \%$ of cases, with a perioperative mortality of $32 \%$ (11). In addition, ruptured AAAs are responsible for $1.3 \%$ of total deaths in $65-85$ year old males in developed countries (10).

The infrarenal aorta is the most common site of AAA formation, and the most favorable anatomical morphology for repair, either via an endovascular approach with EVAR or open surgical repair via infrarenal aortic clamping. Contemporary endovascular technology has made great strides, with EVAR seen as standard care in patients with appropriate anatomy (12).

However, when faced with patient anatomy beyond the standard infrarenal AAA, the endovascular surgeon must modify

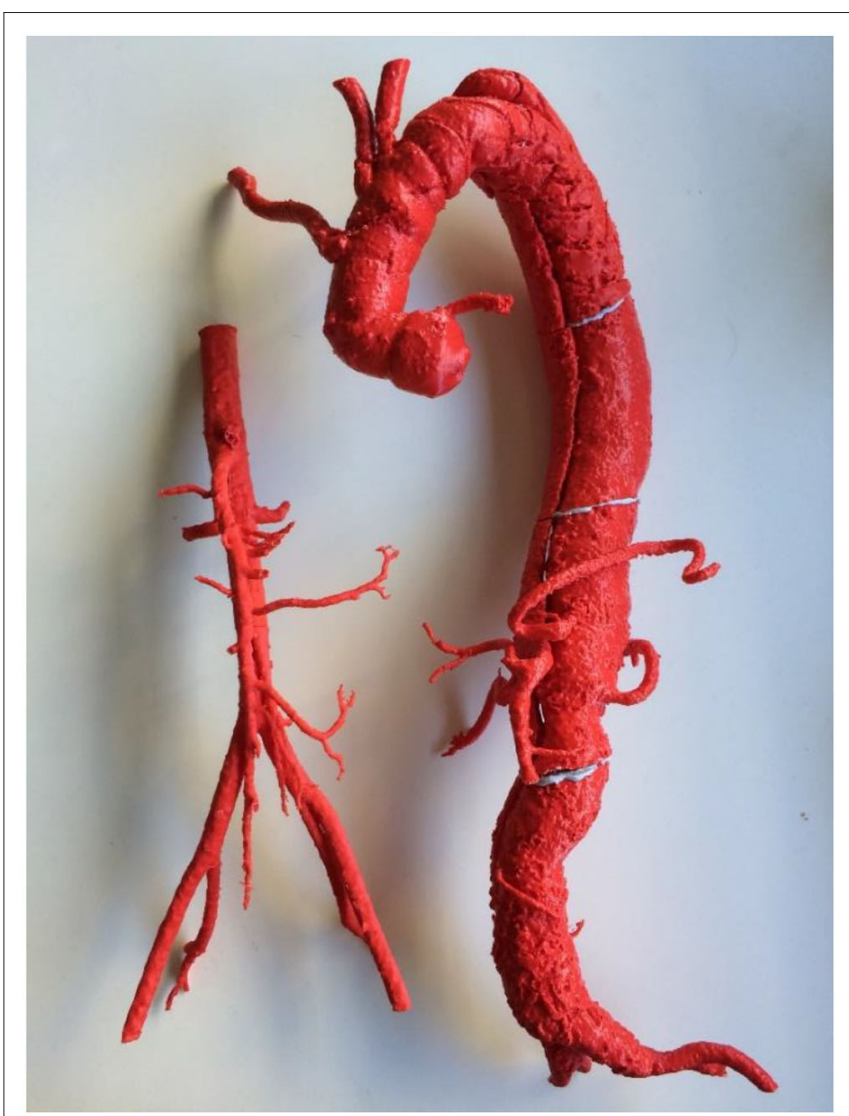

FIGURE 1 | A 3D printed complex Type B aortic dissection for reintervention (right) compared to normal aorta (left). Both models were 3D printed using FDM technology and ABS filament. Note that due to limitations in the size of the print bed, the Type B aortic dissection was printed in four pieces.

his or her approach to these complex cases. If the aneurysm extends to but does not involve the renal artery ostia, it is considered a juxtarenal AAA. If the aneurysm extends further superiorly involving the renal arteries and visceral arteries, it is termed a pararenal or paravisceral AAA. Endovascular repair of these complex anatomies is challenging, requiring branched or fenestrated stent grafts, or may be technically unfeasible with current levels of technology.

\section{Utility of 3D Printed Phantoms in AAA Simulation}

As Vascular Surgery experiences a fundamental shift with increasing endovascular and decreasing open repairs, the reach of EVAR increasingly extends to patients with complex anatomy outside of the standard infrarenal AAA, adding to the complexity of contemporary AAA repair.

Well-known potential complications of EVAR include endoleak, graft occlusion, migration or infection $(13,14)$, requiring further endovascular or open surgical revision and further costs incurred to the healthcare system. As such, adequate visualization of the patient's unique anatomy, appropriate graft selection, the ability to predict intraoperative difficulties and 

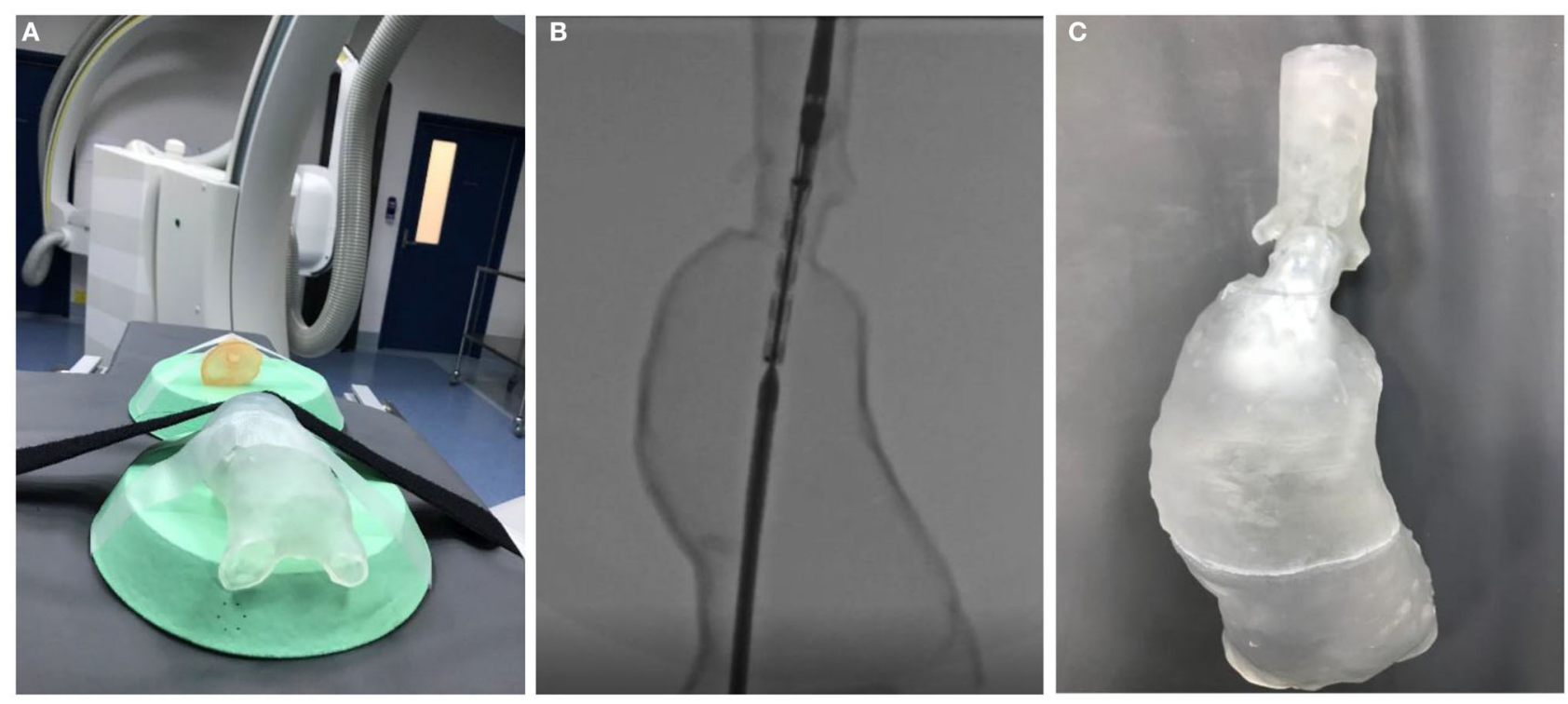

FIGURE 2 | (A) A transparent complex juxtarenal AAA phantom being prepared for simulation of a complex fenestrated EVAR procedure. The model was 3D printed with SLA technology using a transparent resin. (B) The AAA phantom under fluoroscopy. (C) The AAA phantom after deployment of a fenestrated stent graft.

the shape of the graft after deployment are paramount to the improvement of this young technique, and the cost to the public health system. Many of these complications would be mitigated with the opportunity to simulate the proposed procedure and select devices using patient-specific AAA phantoms.

In addition, there is a growing role for $3 \mathrm{D}$ printed EVAR simulators in training the next generation of Vascular Surgeons (7). The benefits of simulation in procedural training have been well-described in the Vascular Surgery literature $(15,16)$. Simulation allows for a "dry run," improving trainee confidence in procedures. It provides an opportunity for participants to apply theory into practice, and to gain experience that would otherwise potentially put patients at risk, particularly in emergency situations. In addition, simulation provides an environment where all members of the team can learn with and from one another, with the opportunity for debriefing and reflection.

Duran et al. reported an improvement in self-reported confidence levels amongst Vascular trainees afforded access to simulation, with $86 \%$ of trainees surveyed supportive of simulation training (17). Simulation accelerates the acquisition of psychomotor skills, procedural understanding, and facilitates assessment of proficiency $(18,19)$. Specific to EVAR, Vento et al. confirmed that simulation objectively improved the competence of trainees in performing EVAR, with reductions in total procedure time, total fluoroscopy time, time for contralateral gate cannulation, and volume contrast used when compared to the control group (20).

Endovascular techniques are evolving at a remarkable rate. Combined with decreased training hours and the unstructured nature of opportunistic on-the-job training via the traditional Halstedian apprenticeship model, simulation-based procedural training is a promising avenue. The contemporary challenge of lack of procedural exposure is further compounded by improvements in non-invasive vascular imaging techniques, reducing the opportunities for trainees to perform diagnostic angiograms in order to gain essential wire and catheter handling skills (18). Simulation provides a solution by offering an avenue to learn the key steps required in common as well as more advanced procedures under the supervision of a surgical educator.

\section{Study Aims}

3D printed vascular phantoms have a growing role in the pre-surgical simulation and training of complex endovascular procedures. While there is growing interest in the topic, lack of familiarity with $3 \mathrm{D}$ printing technology has resulted in slow uptake.

In developing this methodology, our aim was to create inexpensive vascular phantoms with optimal anatomical, haptic and fluoroscopic fidelity. Through iterative prototyping, we have improved upon the workflow, particularly with regards to speed and cost to ensure that initial investment would not pose a barrier to interested departments.

We demonstrate this workflow using AAA phantoms as an example of how CT angiograms acquired as part of standard care can be converted into patient-specific 3D models. Our work has occurred at 3D Med Lab, Australia’s first 3D printing laboratory in a public hospital setting. This work has resulted in hollow 3D printed thoracoabdominal aortas with branches which allow for realistic simulation under fluoroscopy.

These models, despite current material limitations, successfully mimic the cannulation and deployment challenges encountered during live endovascular surgery (Figure 2). As dimensional and representational material validity is improved, these AAA phantoms have the potential to serve as a powerful 

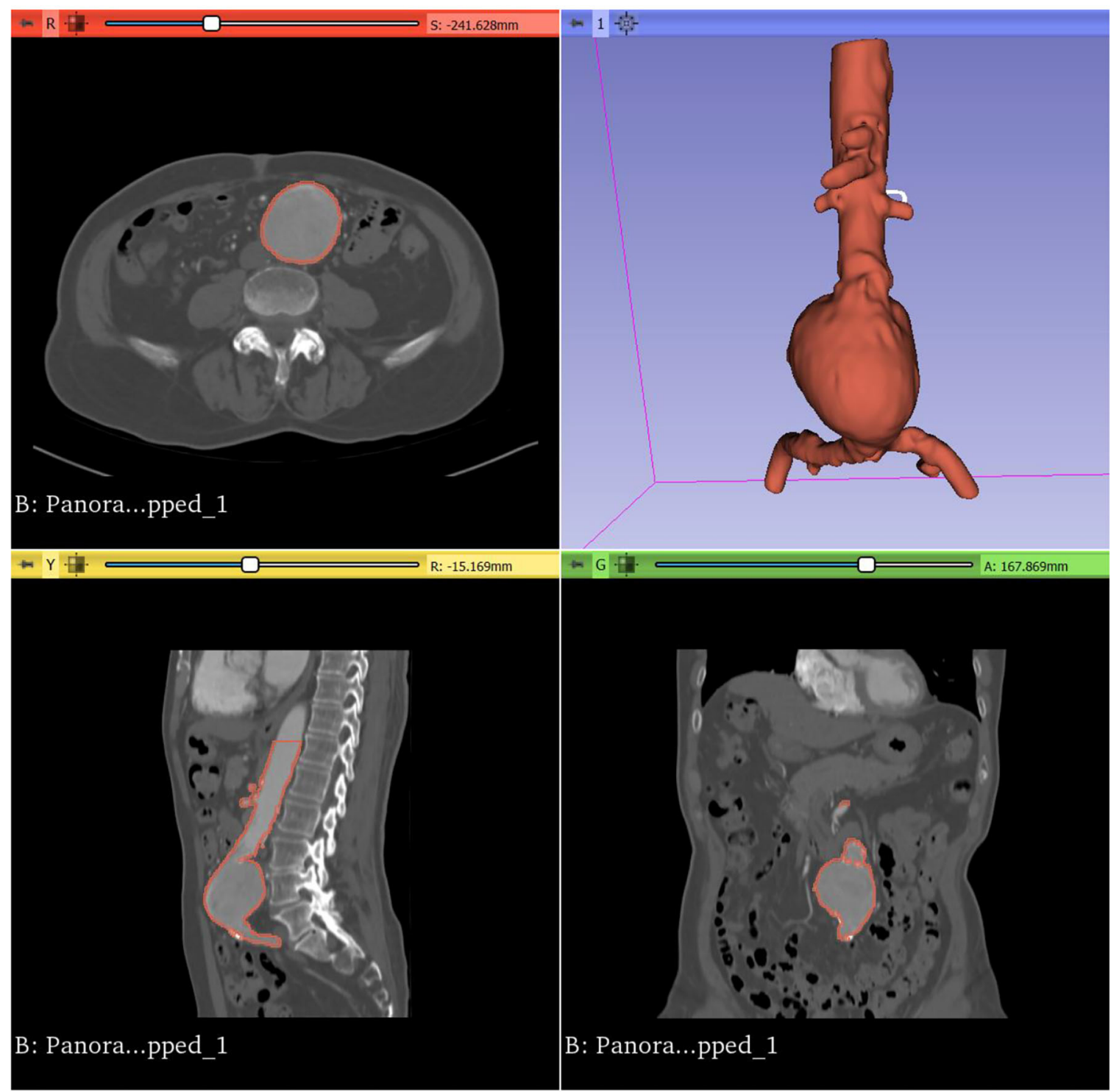

B: Panora...pped_1

R: $-15.169 \mathrm{~mm}$

FIGURE 3 | Surface model of an infrarenal AAA viewed in 3D Slicer.

adjunct to how complex EVAR cases are planned. In addition, as these models do not degrade, they serve as a valuable tool to simulate EVAR for vascular trainees, as well as to counsel patients as part of the therapeutic relationship.

We seek to inform on the readiness of current levels of $3 \mathrm{D}$ printing technologies for the vascular proceduralist, and the feasibility of implementing 3D printing in the hospital setting. A readily reproducible, descriptive methodology for the creation of vascular phantoms has yet to be described in the literature, leading many groups to rely on commercial third parties to create and 3D print their vascular models. Not only would $3 \mathrm{D}$ printed vascular phantoms be more economical to produce within the health service setting, it allows for translation to the angiography suite with the efficiency that surgeons are accustomed to.
In summary, our aim was to develop a low cost, low complexity, CT angiogram to $3 \mathrm{D}$ printed vascular phantom workflow that could be easily adopted by other groups using open source segmentation packages and inexpensive, commercially available $3 \mathrm{D}$ printers.

\section{MATERIALS AND EQUIPMENT}

\section{Segmentation Software}

There is an ever-expanding selection of medical image processing software available, both commercial and open source. Commercial software utilized by groups in the literature include Mimics (version 23.0; Materialize NV, Leuven, Belgium, 2020), InVivoDental (version 6.0; Anatomage, San Jose, CA, 2020), OnDemand3D (APP version 1.0; CyberMed Inc, Seoul, 

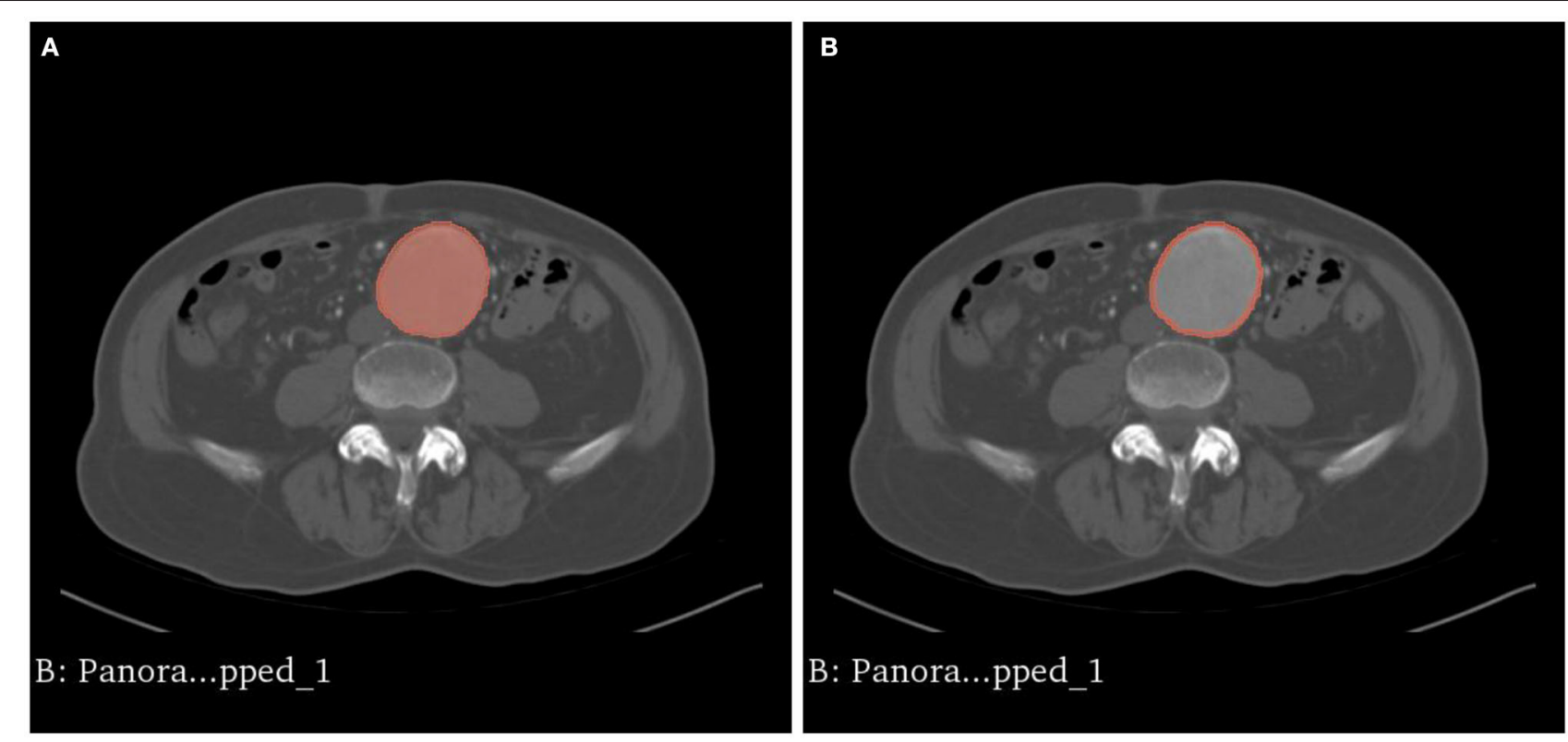

FIGURE 4 | (A) The intraluminal contrast within the AAA is highlighted. (B) The model is dilated outwards to approximate the external surface of the AAA, resulting in a hollow phantom.

Korea, 2020), and OsiriX Imaging Software (version 11.0; Pixmeo, Geneva, Switzerland, 2020). However, licensing fees commence at thousands of dollars, which can be challenging for surgical units to justify at the outset.

Open source software such as 3D Slicer (version 4.11; Harvard, US, 2020), and ITKsnap (version 3.6; Pennsylvania, US, 2020) present accessible alternatives. Most of our group's experience has been with the open source software $3 \mathrm{D}$ Slicer (21), a platform for the analysis and visualization of medical images, available for download at https://www.slicer.org/ (Figure 3). The user interface of 3D Slicer is modular in nature, with powerful plug-in capabilities for additional algorithms and applications.

\section{D Printing Technology}

The most readily available $3 \mathrm{D}$ printing technologies for the vascular proceduralist include those accessible via university links, or those sufficiently affordable and compact to be located within the hospital setting. The most common 3D printing modalities include Fused Deposition Modeling (FDM), Stereolithography (SLA) and Inkjet techniques.

FDM is the most accessible 3D printing technique for those interested in experimenting with the technology, retailing for as little as a few hundred dollars. The technology involves the building of layers via the extrusion of heat-softened polymers. Common materials include the rigid thermoplastics Acrylonitrile Butadiene Styrene (ABS), Polylactic Acid (PLA), as well as flexible thermoplastics such as Thermoplastic Polyurethane (TPU) and Thermoplastic Elastomer (TPE), soft, rubber-like filaments. FDM has been used to 3D print surgical guides (22),

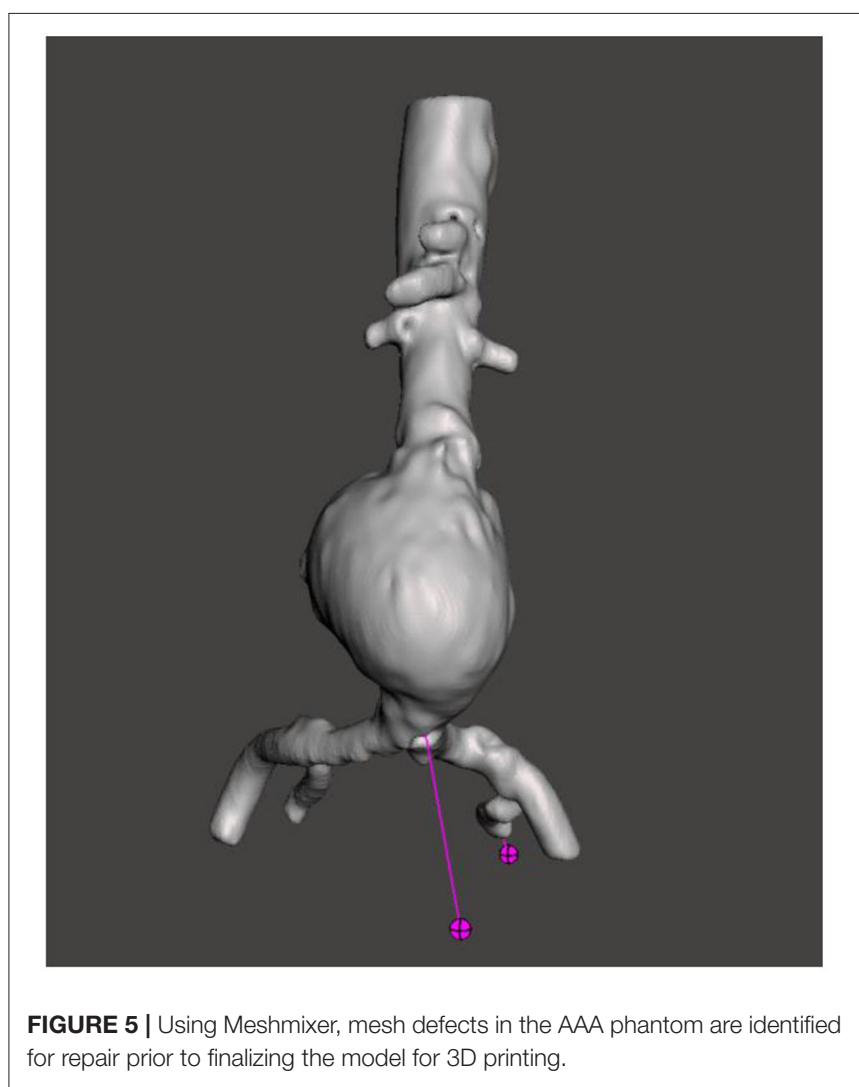

patient-specific anatomical models for presurgical simulation (23), and even patient-tailored pharmacotherapeutics at individualized doses (24). 


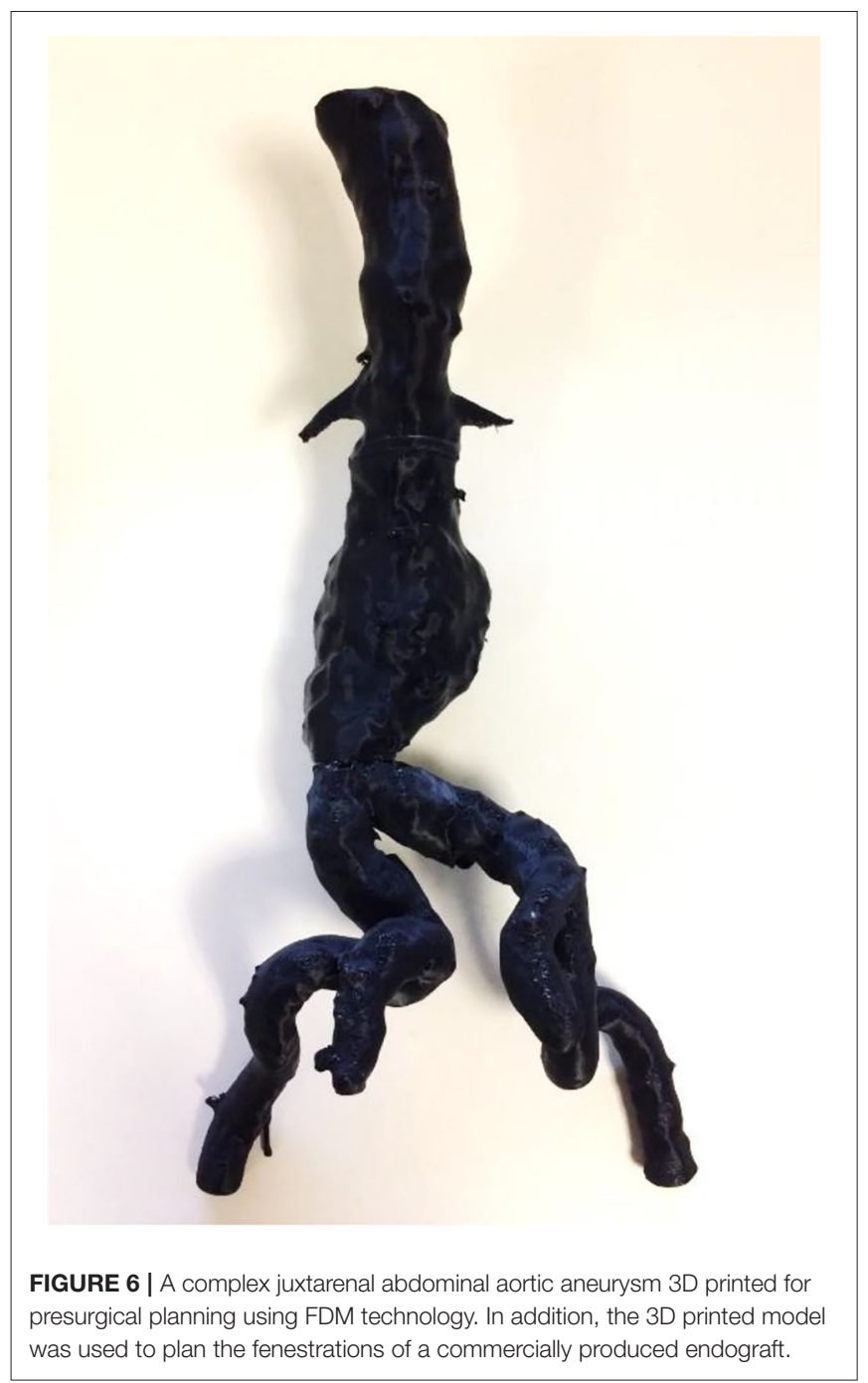

SLA utilizes an ultraviolet laser, which is selectively scanned over a vat of photo-active photopolymer, curing and solidifying specified areas on the surface of the liquid. As the process continues, the final object is built up layer by layer. Thus far, SLA has been used for anatomical modeling in presurgical simulation (25) and training (26), and in the creation of scaffolds for tissue engineering (27).

Inkjet 3D printing is an extension of the conventional twodimensional paper printing technique. Hundreds of microscopic nozzles selectively deposit droplets of photopolymer one layer at a time, which are flash cured using a UV lamp. Retailing for hundreds of thousands of dollars, inkjet printing is the most expensive of the $3 \mathrm{D}$ printing techniques described, requiring collaboration with academic centers at the outset until its expense can be justified. This technique allows for multiple anatomical structures to be $3 \mathrm{D}$ printed in one piece, allowing for basic discrimination between tissues for the purposes of simulation $(28,29)$.

We provide interested vascular proceduralists with examples of aortic phantoms $3 \mathrm{D}$ printed with each of these technologies,

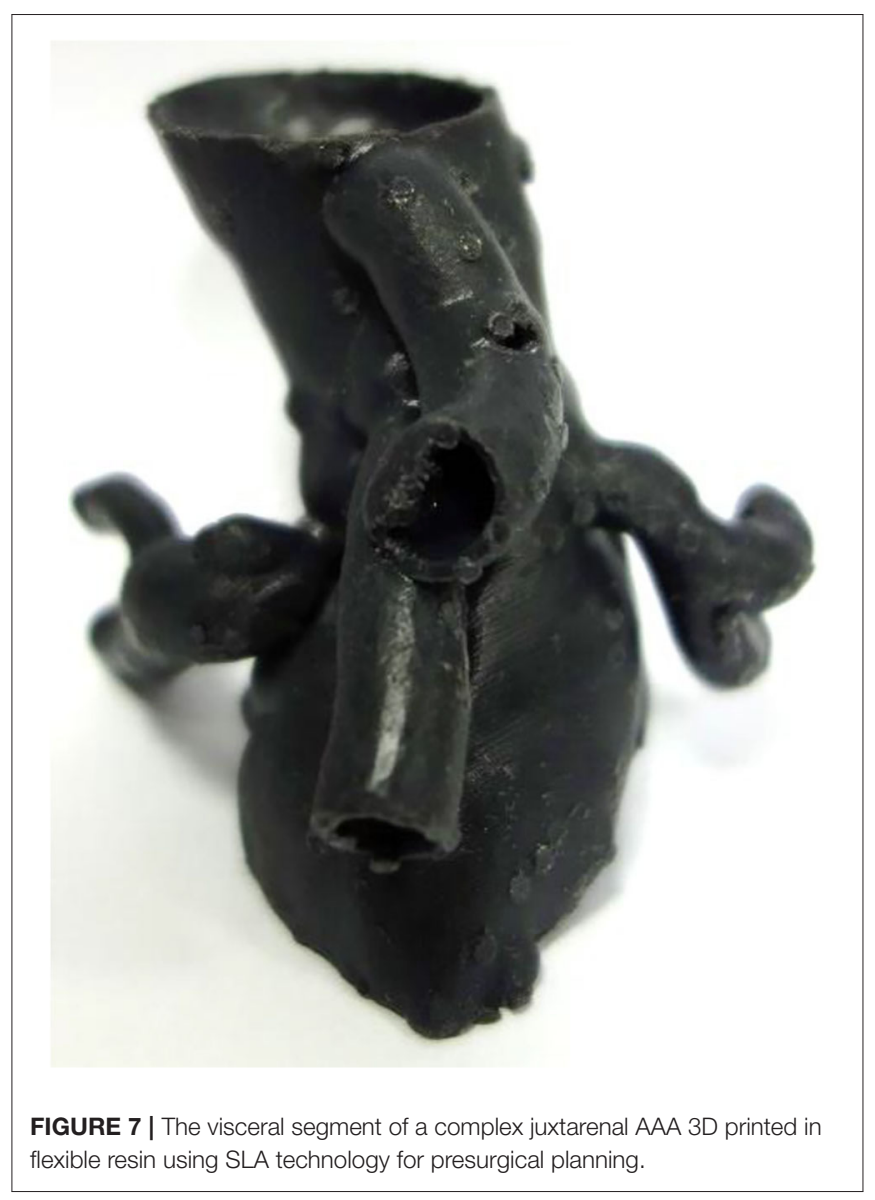

as well as our experiences regarding their suitability for common applications (Figures 6-8).

\section{METHODOLOGY}

We describe our methodology for isolating regions of patient vasculature to generate surface models for 3D printing, however the vascular models generated can equally be viewed on a tablet, through a Virtual Reality headset, or projected onto a screen for viewing.

Using 3D Slicer, CT aortograms were converted to representative patient-specific AAA models. The models were then prepared for printing using another open source computer aided design (CAD) software, Meshmixer (version 3.5, Autodesk, California, US, 2020), and 3D printed using a variety of $3 \mathrm{D}$ printing techniques in order to assess their suitability as aortic phantoms (Figures 4, 5). Depending on the 3D printing technology used, these models cost AUD\$20-1,000 and were produced in $12-48 \mathrm{~h}$.

\section{Image Acquisition}

CT aortograms acquired as part of AAA surveillance or preoperative workup are an ideal starting point for this workflow, with intraluminal contrast greatly facilitating the isolation of 


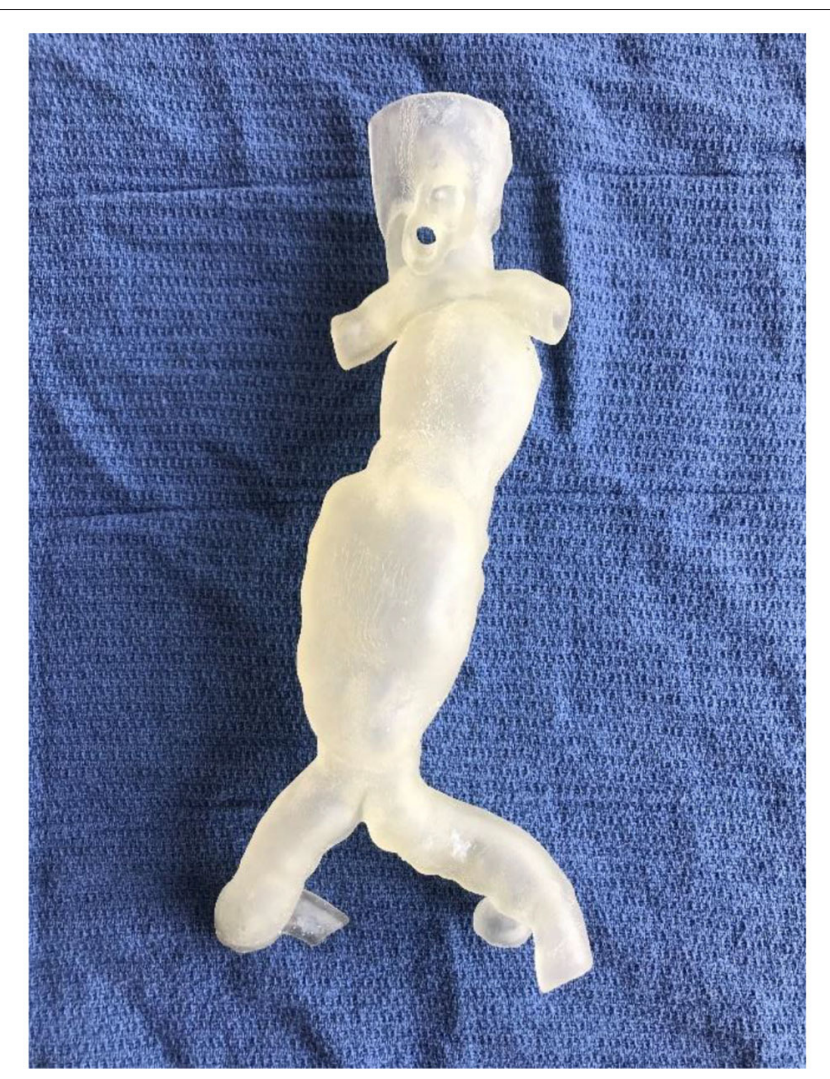

FIGURE 8 | A flexible, hollow, translucent AAA phantom 3D printed using Inkjet technology.

vascular anatomy. Due to its increasing availability and everimproving speed and quality, non-invasive CT angiography has become the conventional imaging modality for visualizing vascular anatomy and pathology (30). The accuracy of image processing is highly dependent upon the quality and resolution of the original CT imaging, with slice thicknesses $<1 \mathrm{~mm}$ yielding superior results.

Although not the modality of choice at our center, Magnetic Resonance Angiography (MRA) presents an ideal alternative, likewise due to the presence of intraluminal contrast. B-mode ultrasound has also been utilized with success in the literature in $3 \mathrm{D}$ printing arteriovenous fistulas (31), however ultrasound as a modality is more heavily operator dependent, less readily available in the clinical setting due to staffing demands, and cannot be applied to all vascular territories. A key concept is that the imaging protocol which best visualizes the vascular anatomy for surgical planning will similarly result in the best imaging for $3 \mathrm{D}$ printing.

\section{Generating 3D Models From CT Angiograms}

Generating 3D models from patient CT angiography is performed via the process of image segmentation, which involves partitioning an image into multiple segments for meaningful analysis. In the context of CT aortograms, a contrast-enhanced AAA and its branches are efficiently isolated from the surrounding soft tissue by leveraging the concept of Hounsfield Units.

Using the Segment Editor module, CT aortogram datasets were automatically segmented using the Threshold function. The Threshold tool divides the CT angiography dataset into two segments, based on the Hounsfield Unit range selected. This labels the voxels, or three-dimensional pixels, as either colored or uncoloured, covering areas of the selected intensity values throughout all slices of the CT angiogram.

Segmentation via thresholding is performed based on intensity values alone. Hence the process, whilst automated, may result in artifact, or unwanted areas that have been highlighted due to their similar density to the region of interest. These can be removed using the Save Island function. Save Island retains the selected anatomy and removes disconnected voxels with the same intensity.

\section{Creating a 3D Model}

In $3 \mathrm{D}$ Slicer, a preview of the segmented AAA can be visualized in the $3 \mathrm{D}$ viewing screen by toggling View $3 \mathrm{D}$. For the purposes of EVAR planning, the AAA can be cropped superior to the visceral segment of the aorta, and inferior to the bifurcation of the common iliac arteries by using the Scissors function. The Hollow function converts the external surface of the intra-luminal contrast into the internal surface, growing a wall of the specified thickness around it, preserving the diameter of the lumen. Once the process is complete, the AAA model is Exported to be prepared for $3 \mathrm{D}$ printing. The AAA model is by default saved in Visualization ToolKit (VTK) format. This is best converted to Standard Triangle Language (STL) format which is compatible with 3D printers and Computer Aided Design (CAD) software.

\section{Preparing the AAA Phantom for 3D Printing}

Depending on the presurgical simulation intended, or the lesson plan in mind, the model can be further modified using CAD software prior to $3 \mathrm{D}$ printing. The vascular phantom can be made modular to introduce increasing levels of complexity, or the inflow and outflow modified to allow compatibility with a fluid circuit. For these purposes, Meshmixer (version 3.5, Autodesk, California, US, 2020) is the open source CAD software favored by many groups in the literature, due to a useful feature in the Analysis menu. The Inspector tool allows the user-friendly Auto Repair of defects in the model prior to $3 \mathrm{D}$ printing.

\section{D Printing the AAA Phantom}

Once the STL file is ready for $3 \mathrm{D}$ printing, it is loaded into the proprietary $3 \mathrm{D}$ printing software associated with the $3 \mathrm{D}$ printing machine and printed. Phantoms produced using the three main 3D printing modalities, Fused Deposition Modeling (FDM), Stereolithography (SLA) and Inkjet are described below in our Results. 

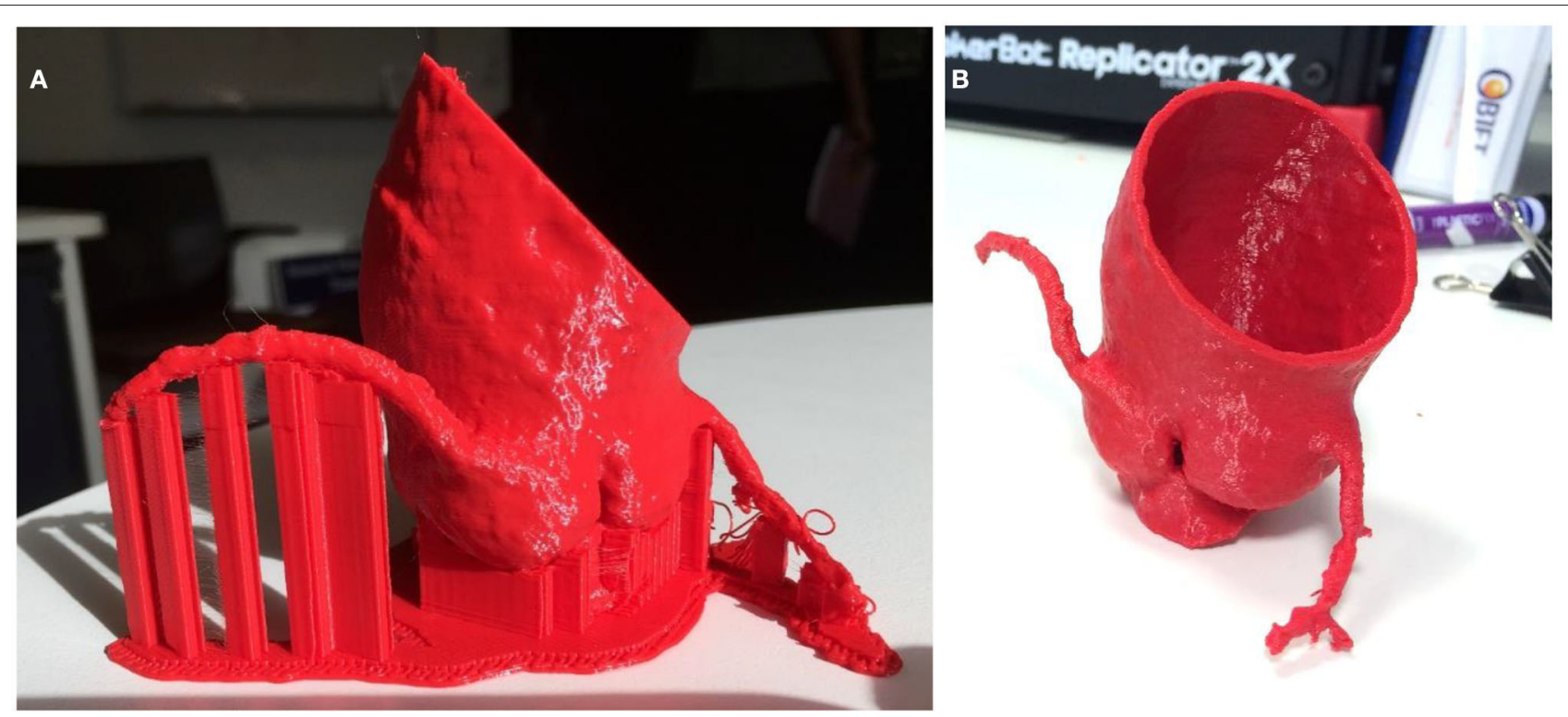

FIGURE 9 | Aortic root model 3D printed in ABS using FDM technology (A) prior to removal of supports (B) with supports removed.

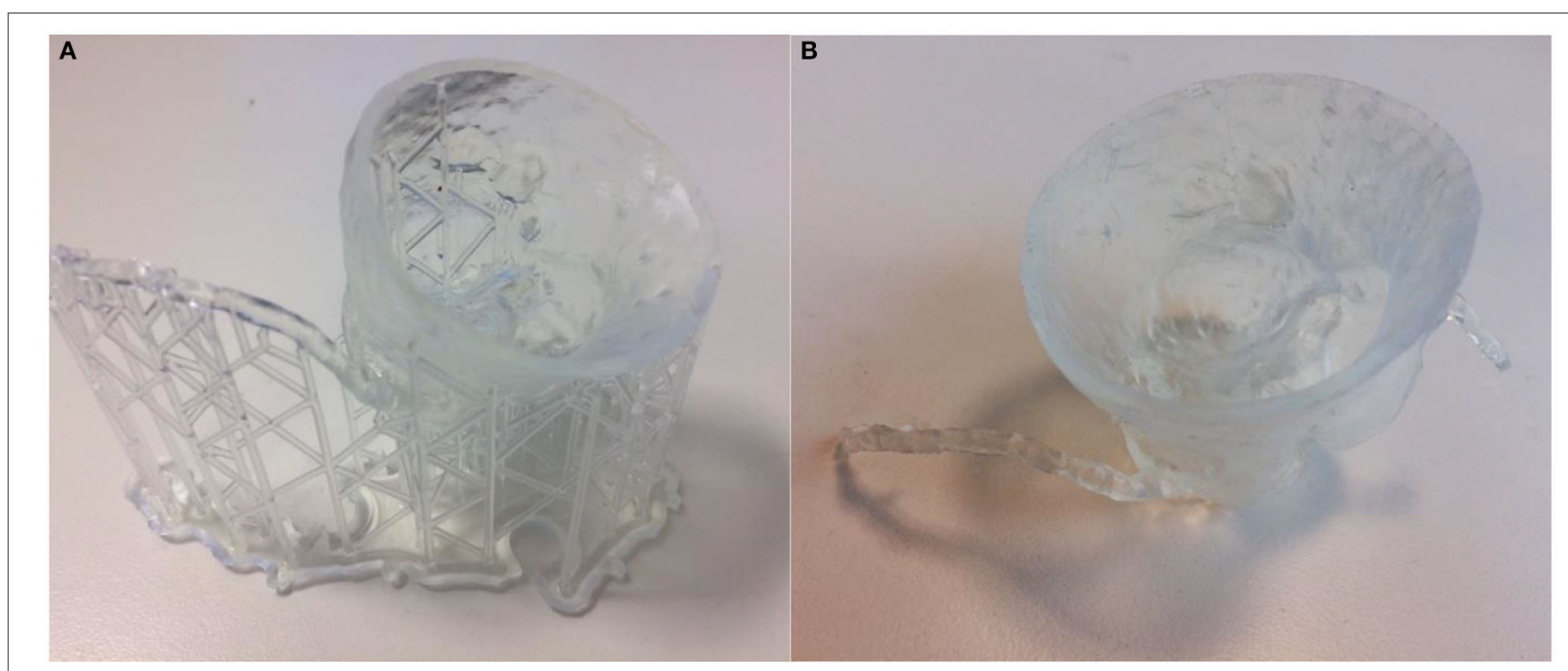

FIGURE 10 | Same aortic root model as previous 3D printed in transparent resin using SLA technology (A) prior to removal of supports (B) with supports removed.

\section{RESULTS}

In our experience, 3D printed AAA phantoms are of limited utility in the presurgical planning of standard infrarenal AAA cases. However, they have influenced surgical decision making and device selection in complex cases. In addition, these models have been $3 \mathrm{D}$ printed and demonstrated to be useful in a variety of settings, including patient education and engagement, surgical and anatomical education, as well as intraoperative visualization.

There remains room for improvement in the manufacturing of these models, in particular for greater cost efficiency and material properties mimicking those of a diseased aorta as we seek to create a AAA phantom with optimal anatomical, haptic and fluoroscopic fidelity.

\section{Fused Deposition Modeling (FDM)}

FDM is an accessible avenue to begin $3 \mathrm{D}$ printing vascular phantoms. There is a large variety of makes and models available on the market, with our group having experience with the Makerbot Replicator 2X (Stratasys, Minnesota, USA), Flashforge Creator Pro (Zhejiang, China), Prusa I3 MK3S (Prague, Czech Republic), and Ultimaker S5 (Utrecht, Netherlands). These 


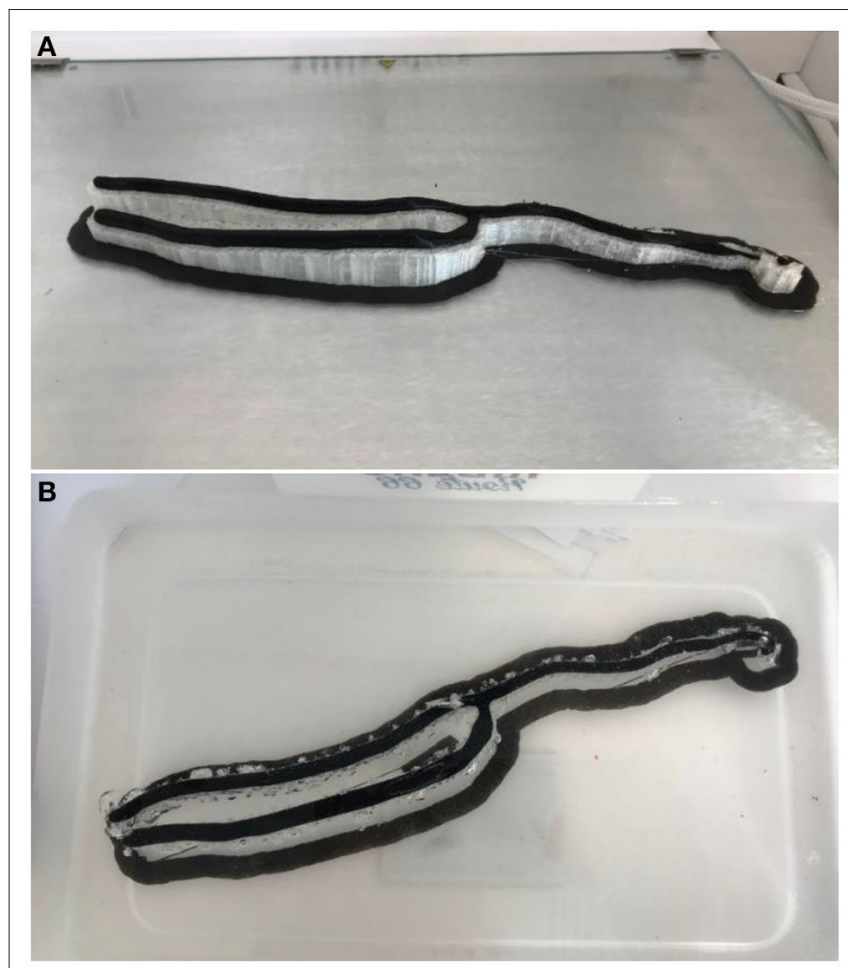

FIGURE 11 | (A) A 3D printed brachial artery model prior to removal from the print bed, with supports in situ. (B) The same model in a water bath to dissolve the PVA supports.

hobbyist $3 \mathrm{D}$ printers retail in the range of hundreds to thousands of dollars, making them an inexpensive option to begin exploring the technology. Professional tier FDM 3D printers are more reliable and require less maintenance.

The machines consume inexpensive thermoplastics, retailing from $\$ 30-80$ for a kilogram, which equates to $\$ 10-20$ per AAA model. Although transparent thermoplastics exist, the resolution of FDM technology and the layered deposition results in at best a translucent end product, as reported by Chung et al. (32). Depending on the size of the AAA being printed, FDM machines require $24-48 \mathrm{~h}$ to $3 \mathrm{D}$ print the final product, with additional time required for support structures to be manually removed.

\section{Stereolithography (SLA)}

SLA printers retail in the range of thousands of dollars. Most of our experience has been with the Formlabs Form 2 (Somerville, Massachusetts, USA) SLA printer. The benefit of the SLA printer over FDM technology is in its greater resolution, allowing for the creation of transparent $3 \mathrm{D}$ printed AAA models which allow the trajectory of devices to be visualized during simulation. $1 \mathrm{~L}$ of clear or opaque resin retails for $\$ 150$, equating to roughly $\$ 50-$ 100 per model. Despite the slightly higher cost, we have come to rely on the SLA printer due to its lower print failure rate and ability to create transparent models.

In addition, Formlabs carry an autoclavable dental resin which has received FDA approval to be autoclaved. This has resulted in early work in the literature of 3D printed AAA visceral segment models for the planning of fenestrated physician modified stent grafts on the sterile back table (33).

\section{Inkjet}

Inkjet printers produce multicolored, multi-material models with variable shore hardness. Our group have used the Object500 Connex3 Polyjet (Stratasys, Minneapolis, USA), the Stratasys J750 (Stratasys, Minneapolis, USA), and the Projet 3500 3D printers (3D Systems Corporation, Rock Hill, USA) to produce flexible, translucent aortic phantoms. Due to the greater size of the print bed on these commercial 3D printers, even most large AAAs can be $3 \mathrm{D}$ printed in a single piece, costing $\$ 700-1,000$ in materials.

At the outset, Inkjet printers require a significant financial investment or collaboration with an academic center when building a hospital-based $3 \mathrm{D}$ printing service. For example, the Stratasys J750 retails for $\$ 600,000$, and a $1 \mathrm{~kg}$ cartridge of the resin required for $3 \mathrm{D}$ printing costs $\$ 2,000$.

\section{Postprocessing}

Regardless of the 3D printing technique, FDM, SLA, and Inkjet $3 \mathrm{D}$ printed phantoms require postprocessing prior to use, and the removal of support material required to support the weight of the object during the $3 \mathrm{D}$ printing process. Disposal metal scissors and forceps allow for the removal of support material with accuracy and control. This process typically requires a few minutes of manual removal, and up to $20 \mathrm{~min}$ for more intricate models (Figures 9-10).

Dissolvable support material is an alternative, with Polyvinyl Acetate (PVA) support material available for dual-extrusion FDM machines, which is readily dissolved in water. Chemical solvents are required to dissolve the large amount of supports present on Inkjet 3D printed models (Figure 11). This is preferable to water jetting the support material, which risks damaging intricate anatomy, particularly when producing flexible models. A disadvantage of soluble support material is the overnight wait for supports to dissolve, adding to the production time of the workflow.

Depending on the $3 \mathrm{D}$ printer used, the AAA may need to be sectioned into several parts to fit the available volume for printing. In particular, conventional hobbyist FDM and SLA 3D printers are limited by small print beds, with larger thoracoabdominal aneurysms required to be $3 \mathrm{D}$ printed in pieces and joined together with epoxy resin.

\section{Performance Under Fluoroscopy}

FDM, SLA and Inkjet 3D printed AAA phantoms are equally visible under fluoroscopy, allowing for another level of realism to be added to the simulation task. Despite the fact that all three 3D printing techniques performed equally from a visual perspective, due to the resolution of FDM technology which features building melted layers of plastic to create the final product, the grooves between layers were haptically perceptible when traversed by wires (Figure 12). While superior to conventional CT angiography or workstation $3 \mathrm{D}$ reconstructions, uneven ridges have the potential to affect the trajectory of guidewires 

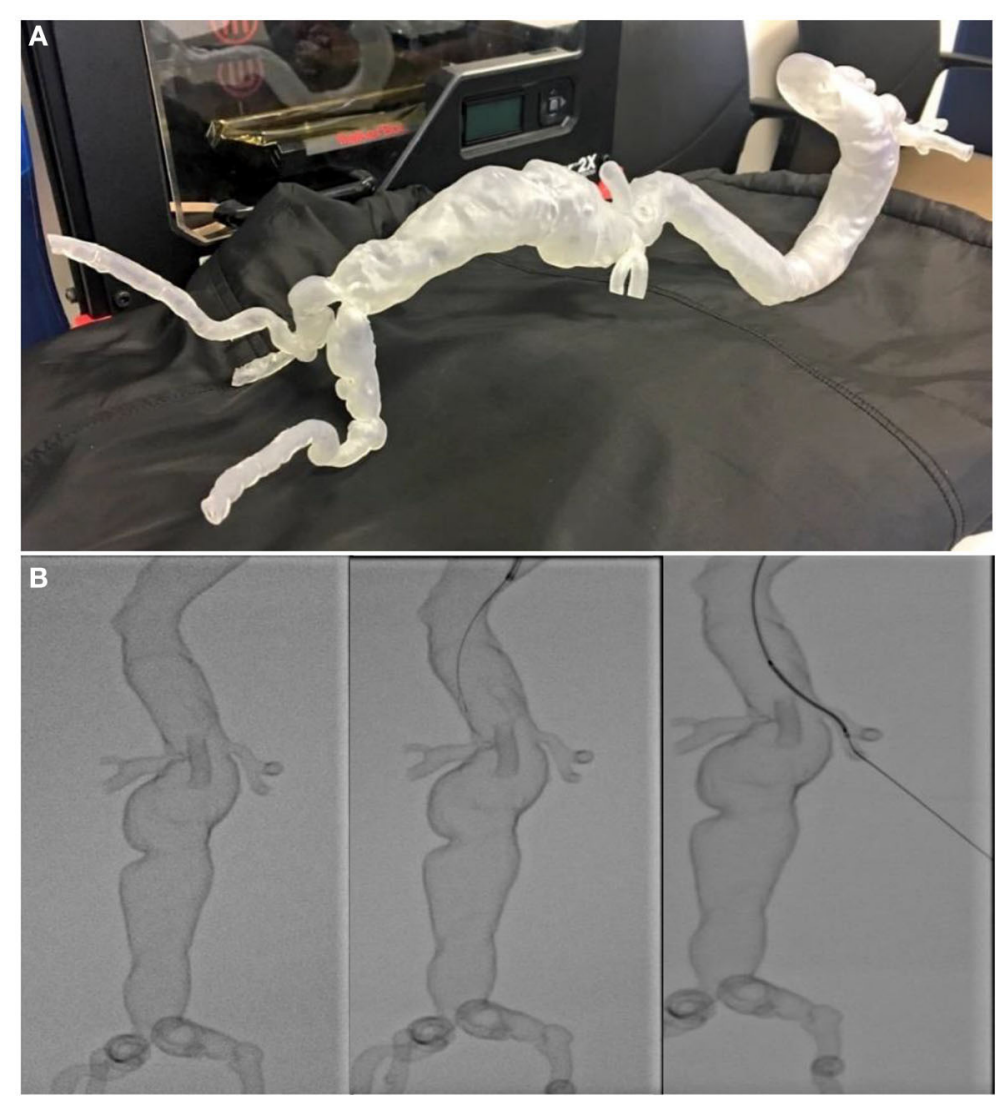

FIGURE 12 | (A) A hollow transparent complex thoracoabdominal aneurysm phantom 3D printed using SLA technology. (B) Stages of the phantom being cannulated under fluoroscopy for interventional planning. This "pre-flight" simulation was invaluable in predicting navigational difficulties, with the trajectory of guidewires and devices matching what was encountered during live surgery.

and devices during preoperative simulation. In addition, we have found that the lack of compliance in rigid $3 \mathrm{D}$ printed materials adds an additional level of difficulty when traversing tortuous iliac arteries.

\section{DISCUSSION}

AAA phantoms $3 \mathrm{D}$ printed using a variety of $3 \mathrm{D}$ printing technologies, despite material limitations, successfully mimic the cannulation and deployment challenges encountered during live endovascular surgery. As dimensional and representational material validity improves, they have the potential to serve as a powerful adjunct to how complex EVAR cases are planned.

\section{Comparison of 3D Printed AAA Phantoms to Other Vascular Territories}

3D printed AAAs, and other large vascular phantoms involving the aorta, result in greater challenges with regard to efficient production. Unless access to commercial 3D printers are readily accessible, 3D printed AAAs must often be produced in pieces and joined together prior to use, due to limitations on the size of the print bed on hobbyist machines.
In the case of simulating procedures in smaller vascular territories such as the Circle of Willis or Internal Carotid Artery it may be wiser to create a negative of the anatomy of the interest contained within a solid 3D printed block. From our experience in these smaller vascular territories this creates a more durable result in these small diameter vessels which are more prone to moving or breaking during simulation.

\section{Anatomical Accuracy of 3D Printed Phantoms}

We have outlined the workflow required to produce 3D printed vascular phantoms, with each step introducing a potential avenue for error during the imaging, segmentation, or 3D printing phases. It is evident that further work validating the accuracy of $3 \mathrm{D}$ printed models for surgical simulation is warranted. As with all new devices and techniques, surgeons are accustomed to the circumspect application of new technologies to meet the needs of each individual patient.

\section{Image Acquisition}

Patient-specific anatomical models are as accurate as the imaging from which they originate. A useful principle is that the imaging modality that best visualizes the anatomy for conventional 


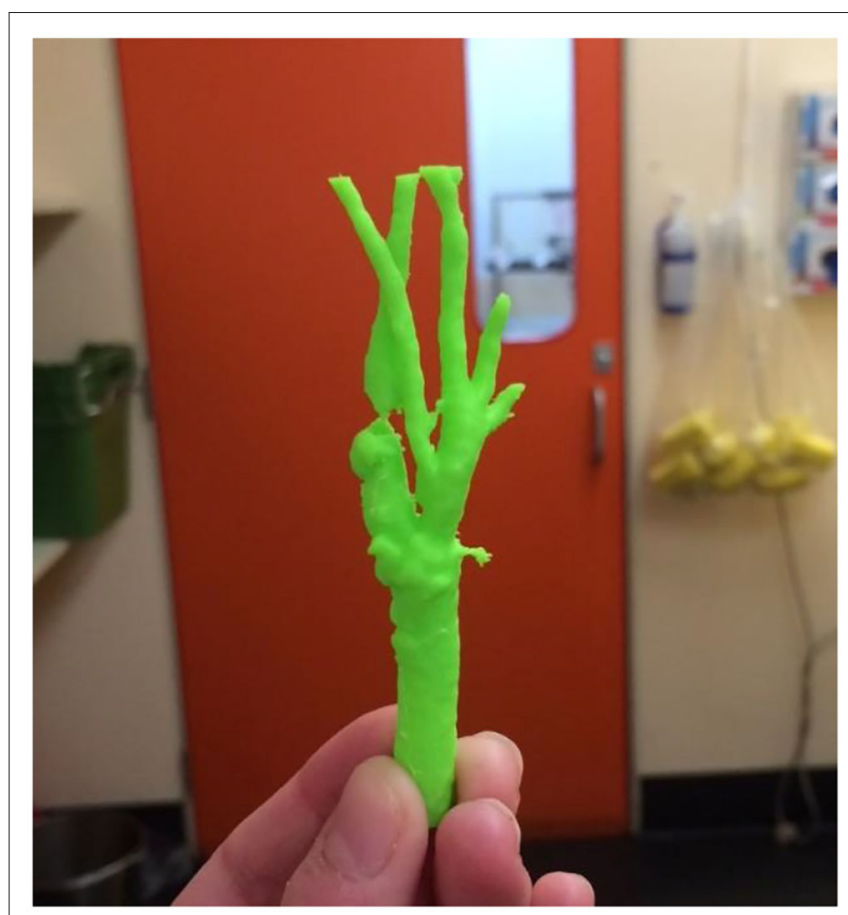

FIGURE 13 | A solid 3D printed internal carotid artery used to plan carotid endarterectomy.

surgical planning will produce the most detailed anatomical model. Imaging modalities are ever improving, with these improvements benefiting patients regardless of whether an anatomical model is 3D printed (Figure 13). In the vascular territories, optimizing vascular imaging requires the mitigation of motion artifact, and sufficient contrast discrimination and resolution.

For example, dual-energy CT angiography would allow for better contrast discrimination of intravascular calcium from contrast, as well as improving temporal resolution in both peripheral and aortic vascular territories (34, 35). Similarly, electrocardiographic-gated CT coronary angiography allows for the reduction of motion artifacts in valvular heart disease (36), leading to a superior 3D printed anatomical model.

It is evident that as $3 \mathrm{D}$ printing becomes more grounded in conventional surgical practice, improving current imaging protocols in order to optimize the final $3 \mathrm{D}$ printed product is a necessary area of further study.

\section{D Modeling}

Medical image processing software is the next step in the workflow that has a potential to introduce error. Kang et al. compared four of the most readily available commercial software options, InVivoDental (version 5.0; Anatomage, San Jose, CA, 2015), Mimics (version 14.0; Materialize NV, Leuven, Belgium, 2015), OnDemand3D (APP version 1.0; CyberMed Inc, Seoul, Korea, 2015), and OsiriX Imaging Software (version 3.7; Pixmeo, Geneva, Switzerland, 2015) in the creation of craniofacial models (37), determining that InVivoDental was most accurate in producing $3 \mathrm{D}$ models from $\mathrm{CT}$ imaging.
All four segmentation software packages were accurate at the voxel or subvoxel level; however, there were statistically significant differences in all anatomical regions between the four software packages tested. Kang et al. were not able to access the proprietary coding of these commercial software packages, hence the study was unable to apply the same standardized parameters across the board, limiting direct comparison. Despite this, the fact that all models were accurate to the level of individual voxels was a reassuring finding.

\section{D Printing}

There is currently no gold standard for validating the accuracy of patient-specific anatomical models produced by $3 \mathrm{D}$ printing. However, early work from multiple groups suggests the accuracy of $3 \mathrm{D}$ printed models are within acceptable limits for presurgical planning (38-41).

Hazeveld et al. compared the accuracy of different 3D printing technologies (42). Given what is known about the accuracy of each 3D printing technology, the study confirmed that dimensional error was lowest for Inkjet 3D printing, followed by SLA, and finally FDM. Specific to 3D printed vasculature, Takao et al. determined splenic artery aneurysms 3D printed using FDM printers to be highly precise and accurate, with the cross-sectional areas amongst the $3 \mathrm{D}$ printed models within $\mathrm{SD}$ $<0.05 \mathrm{~cm}^{2}$ (range 0.00-0.05) (6).

\section{Determining Validity}

The ideal AAA phantom is one that is not only anatomically accurate but boasts high haptic and fluoroscopic fidelity. In developing this workflow, we have sought to produce 3D printed AAAs with the highest possible representational validity within the constraints of a bench to bedside approach, accessible to the hospital vascular proceduralist.

The necessity of each measure of fidelity depends upon its intended application. For example, for the purposes of presurgical simulation and the education of trainees, high haptic and fluoroscopic fidelity are much more important than when a $3 \mathrm{D}$ printed AAA model is used to educate patients. As previously discussed by our group, the validity of a simulation tool can be evaluated in several ways, with a current lack of objective evidence as to how the different measures of validity can be assessed (43). Face validity involves comparing the anatomical and haptic fidelity of a simulator to the current "gold standard" trainers or to performing the procedure in vivo (44). It is a highly subjective, but most common measure used in the medical simulation literature. Predictive validity is a much more objective and desirable goal, and involves an assessment of patient outcomes (44) as a result of the simulation. As previously discussed, there are clinical trials underway exploring if $3 \mathrm{D}$ printed anatomical models for presurgical planning do indeed improve patient outcomes (1), but none on the topic of endovascular intervention.

\section{A Hospital-Based 3D Printing Service}

3D printed anatomical models present a promising new frontier in the planning and simulation of complex surgical procedures. The American Medical Association has recognized the potential 


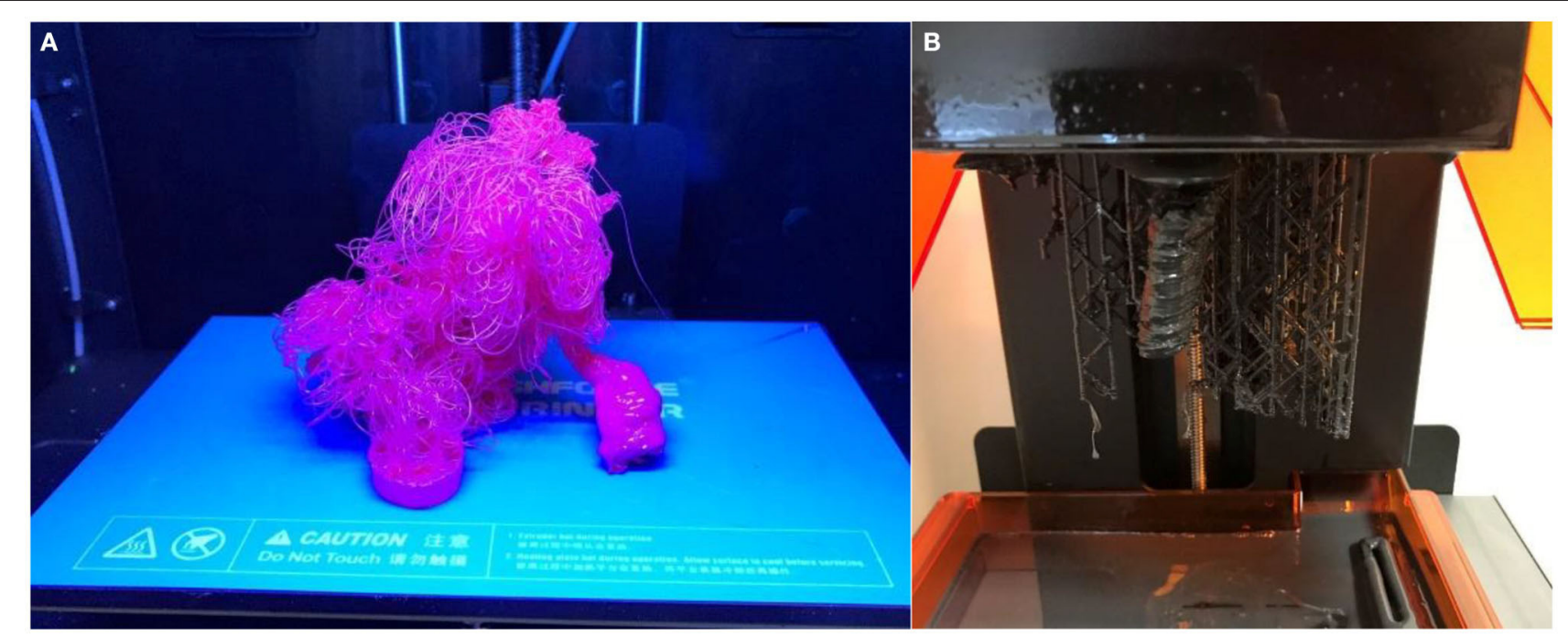

FIGURE 14 | Failed prints on (A) FDM. (B) SLA 3D printers.

of 3D printed anatomical models in presurgical planning and in producing surgical guides, introducing two reimbursement codes last year (45).

The strength of $3 \mathrm{D}$ printing anatomical models when compared to other technologies is its unprecedented accessibility to the vascular specialist, allowing for just-intime manufacturing. When compared to commissioning an external commercial provider, 3D printed vascular phantoms can be translated from bench to bedside in a matter of hours, lowering costs to surgical units and the time required for the model to be shipped to the hospital. In addition, the current COVID-19 pandemic has emphasized the importance of local health services to be self-sufficient with regard to supplies.

However, the centralized manufacturing approach requires local hospitals to invest in the medical image processing, $\mathrm{CAD}$ and $3 \mathrm{D}$ printing hardware and skills outlined in this manuscript in order to access this technology. While this would result in significant cost savings for the anatomical models themselves, with flow-on effects of reduced theater time and blood loss resulting in cost savings to the hospital system, it represents a more substantial initial investment.

\section{Limitations of Current Technology}

3D printing remains a rapidly evolving technology which is becoming increasingly accessible to vascular proceduralists and the general public. With current levels of technology, inexpensive hobbyist $3 \mathrm{D}$ printers are less reliable than their commercial counterparts, particularly when first experimenting with the print orientation of objects. Despite this, they remain an accessible starting point for vascular specialists seeking to begin their foray into the technology (Figure 14). The print failure rate is lessened when printing smaller models, when compared to large vascular phantoms such as thoracoabdominal aneurysms, and when $3 \mathrm{D}$ printers are adequately maintained.

\section{CONCLUSION}

Once the challenges in developing this workflow were overcome, $3 \mathrm{D}$ printed anatomical models have become commonplace in the planning of complex procedures in our Vascular Unit and the broader Department of Surgery. Physical patient-specific models have proven to be a valuable addition to standard imaging, and in the rehearsal and modification of devices prior to surgery. When planning the approach to complex AAAs, hollow flexible models are particularly useful for the rehearsal of endograft insertion and positioning via iliac artery access, and in predicting the trajectory of guidewires and devices.

As $3 \mathrm{D}$ printing technologies become more common, reliable, and cost effective, their use in preprocedural simulation will flourish. In sharing this methodology, we warmly invite comments from others with an interest in $3 \mathrm{D}$ printing in vascular interventional planning in how we can further explore its uses.

\section{DATA AVAILABILITY STATEMENT}

The raw data supporting the conclusions of this article will be made available by the authors, without undue reservation.

\section{AUTHOR CONTRIBUTIONS}

JC-B contributed to the write up, study design, and experiments performed in this manuscript. DB contributed to the write up and design of this manuscript. JC contributed to the write up, design, and supervision of this manuscript. All authors contributed to the article and approved the submitted version. 


\section{REFERENCES}

1. Witowski J, Sitkowski M, Zuzak T, Coles-Black J, Chuen J, Major P, et al. From ideas to long-term studies: 3D printing clinical trials review. Int J Comput Assist Radiol Surg. (2018) 13:1473-8. doi: 10.1007/s11548-018-1793-8

2. Marone EM, Auricchio F, Marconi S, Conti M, Rinaldi LF, Pietrabissa $\mathrm{A}$, et al. Effectiveness of $3 \mathrm{D}$ printed models in the treatment of complex aortic diseases. J Cardiovasc Surg (Torino). (2018) 59:699706. doi: 10.23736/S0021-9509.18.10324-7

3. Taher F, Falkensammer J, McCarte J, Strassegger J, Uhlmann M, Schuch $\mathrm{P}$, et al. The influence of prototype testing in three-dimensional aortic models on fenestrated endograft design. J Vasc Surg. (2017) 65:15917. doi: $10.1016 /$ j.jvs.2016.10.108

4. Leotta DF, Starnes BW. Custom fenestration templates for endovascular repair of juxtarenal aortic aneurysms. J Vasc Surg. (2015) 61:163741. doi: 10.1016/j.jvs.2015.02.016

5. Kono K, Shintani A, Okada H, Terada T. Preoperative simulations of endovascular treatment for a cerebral aneurysm using a patientspecific vascular silicone model. Neurol Med Chir (Tokyo). (2013) 53:34751. doi: $10.2176 / \mathrm{nmc} .53 .347$

6. Takao H, Amemiya S, Shibata E, Ohtomo K. 3D printing of preoperative simulation models of a splenic artery aneurysm: precision and accuracy. Acad Radiol. (2017) 24:650-3. doi: 10.1016/j.acra.2016.12.015

7. Torres IO, De Luccia N. A simulator for training in endovascular aneurysm repair: The use of three dimensional printers. Eur J Vasc Endovasc Surg. (2017) 54:247-53. doi: 10.1016/j.ejvs.2017.05.011

8. Qiao F, Li D, Jin Z, Hao D, Liao Y, Gong S. A novel combination of computerassisted reduction technique and three dimensional printed patient-specific external fixator for treatment of tibial fractures. Int Orthop. (2016) 40:83541. doi: 10.1007/s00264-015-2943-z

9. Coles-Black J, Chao I, Chuen J. Three-dimensional printing in medicine. Med J Aust. (2017) 207:102-3. doi: 10.5694/mja16.01073

10. Sakalihasan N, Limet R, Defawe OD. Abdominal aortic aneurysm. Lancet. (2005) 365:1577-89. doi: 10.1016/S0140-6736(05)66459-8

11. Kniemeyer HW, Kessler T, Reber PU, Ris HB, Hakki H, Widmer MK. Treatment of ruptured abdominal aortic aneurysm, a permanent challenge or a waste of resources? Prediction of outcome using a multi-organ-dysfunction score. Eur J Vasc Endovasc Surg. (2000) 19:190-6. doi: 10.1053/ejvs.1999.0980

12. Calero A, Illig KA. Overview of aortic aneurysm management in the endovascular era. Semin Vasc Surg. (2016) 29:317. doi: 10.1053/j.semvascsurg.2016.07.003

13. Greenhalgh RM, Brown LC, Kwong GP, Powell JT, Thompson SG. Comparison of endovascular aneurysm repair with open repair in patients with abdominal aortic aneurysm (EVAR trial 1), 30-day operative mortality results: randomised controlled trial. Lancet. (2004) 364:8438. doi: 10.1016/S0140-6736(04)16979-1

14. Prinssen M, Verhoeven EL, Buth J, Cuypers PW, van Sambeek MR, Balm $\mathrm{R}$, et al. A randomized trial comparing conventional and endovascular repair of abdominal aortic aneurysms. N Engl J Med. (2004) 351:160718. doi: 10.1056/NEJMoa042002

15. Bismuth J, Donovan MA, O’Malley MK, El Sayed HF, Naoum JJ, Peden EK, et al. Incorporating simulation in vascular surgery education. J Vasc Surg. (2010) 52:1072-80. doi: 10.1016/j.jvs.2010.05.093

16. Widmer LW, Schmidli J, Widmer MK, Wyss TR. Simulation in vascular access surgery training. J Vasc Access. (2015) 16(Suppl. 9):S121-5. doi: 10.5301/jva.5000372

17. Duran C, Bismuth J, Mitchell E. A nationwide survey of vascular surgery trainees reveals trends in operative experience, confidence, and attitudes about simulation. J Vasc Surg. (2013) 58:524-8. doi: 10.1016/j.jvs.2012.12.072

18. Neequaye SK, Aggarwal R, Van Herzeele I, Darzi A, Cheshire NJ. Endovascular skills training and assessment. J Vasc Surg. (2007) 46:105564. doi: 10.1016/j.jvs.2007.05.041

19. Aggarwal R, Black SA, Hance JR, Darzi A, Cheshire NJ. Virtual reality simulation training can improve inexperienced surgeons' endovascular skills. Eur J Vasc Endovasc Surg. (2006) 31:588-93. doi: 10.1016/j.ejvs.2005.11.009

20. Vento V, Cercenelli L, Mascoli C, Gallitto E, Ancetti S, Faggioli G, et al. The role of simulation in boosting the learning curve in EVAR procedures. J Surg Educ. (2018) 75:534-40. doi: 10.1016/j.jsurg.2017.08.013
21. Fedorov A, Beichel R, Kalpathy-Cramer J, Finet J, Fillion-Robin JC, Pujol S, et al. 3D Slicer as an image computing platform for the Quantitative Imaging Network. Magn Reson Imaging. (2012) 30:132341. doi: 10.1016/j.mri.2012.05.001

22. Chana-Rodriguez F, Mananes RP, Rojo-Manaute J, Gil P, Martinez-Gomiz JM, Vaquero-Martin J. 3D surgical printing and pre contoured plates for acetabular fractures. Injury. (2016) 47:2507-11.

23. Scawn RL, Foster A, Lee BW, Kikkawa DO, Korn BS. Customised 3D printing: an innovative training tool for the next generation of orbital surgeons. Orbit. (2015) 34:216-9. doi: 10.3109/01676830.2015.1049367

24. Skowyra J, Pietrzak K, Alhnan MA. Fabrication of extended-release patienttailored prednisolone tablets via fused deposition modelling (FDM) 3D printing. Eur J Pharm Sci. (2015) 68:11-7. doi: 10.1016/j.ejps.2014.11.009

25. Man QW, Jia J, Liu K, Chen G, Liu B. Secondary reconstruction for mandibular osteoradionecrosis defect with fibula osteomyocutaneous flap flowthrough from radial forearm flap using stereolithographic 3dimensional printing modeling technology. J Craniofac Surg. (2015) 26:e1903. doi: $10.1097 /$ SCS.0000000000001456

26. Dhir V, Itoi T, Fockens P, Perez-Miranda M, Khashab MA, Seo DW, et al. Novel ex vivo model for hands-on teaching of and training in EUSguided biliary drainage: creation of "Mumbai EUS" stereolithography/3D printing bile duct prototype (with videos). Gastrointest Endosc. (2015) 81:4406. doi: 10.1016/j.gie.2014.09.011

27. Skoog SA, Goering PL, Narayan RJ. Stereolithography in tissue engineering. $J$ Mater Sci Mater Med. (2014) 25:845-56. doi: 10.1007/s10856-013-5107-y

28. Barbosa MZ, Zylbersztejn DS, de Mattos LA, Carvalho LF. Three-dimensionally-printed models in reproductive surgery: systematic review and clinical applications. Minerva Ginecol. (2019) 71:235-44. doi: 10.23736/S0026-4784.19.04319-3

29. Maddox MM, Feibus A, Liu J, Wang J, Thomas R, Silberstein JL. 3Dprinted soft-tissue physical models of renal malignancies for individualized surgical simulation: a feasibility study. J Robot Surg. (2018) 12:2733. doi: 10.1007/s11701-017-0680-6

30. Yu T, Zhu X, Tang L, Wang D, Saad N. Review of CT angiography of aorta. Radiol Clin North Am. (2007) 45:461-83. doi: 10.1016/j.rcl.2007.04.010

31. Carroll JE, Colley ES, Thomas SD, Varcoe RL, Simmons A, Barber TJ. Tracking geometric and hemodynamic alterations of an arteriovenous fistula through patient-specific modelling. Comput Methods Programs Biomed. (2020) 186:105203. doi: 10.1016/j.cmpb.2019.105203

32. Chung M, Radacsi N, Robert C, McCarthy ED, Callanan A, Conlisk N, et al. On the optimization of low-cost FDM 3D printers for accurate replication of patient-specific abdominal aortic aneurysm geometry. 3D Print Med. (2018) 4:2. doi: 10.1186/s41205-017-0023-2

33. Branzan D, Winkler D, Schmidt A, Scheinert D, Grunert R. 3-Dimensional aortic model to create a fenestrated stent graft for the urgent treatment of a paravisceral penetrating aortic ulcer. JACC Cardiovasc Interv. (2019) 12:793-5. doi: 10.1016/j.jcin.2018.10.024

34. Brockmann C, Jochum S, Sadick M, Huck K, Ziegler P, Fink C, et al. Dualenergy CT angiography in peripheral arterial occlusive disease. Cardiovasc Intervent Radiol. (2009) 32:630-7. doi: 10.1007/s00270-008-9491-5

35. Shaqdan KW, Parakh A, Kambadakone AR, Sahani DV. Role of dual energy CT to improve diagnosis of non-traumatic abdominal vascular emergencies. Abdom Radiol (NY). (2019) 44:406-21. doi: 10.1007/s00261-018-1741-7

36. Manghat NE, Rachapalli V, Van Lingen R, Veitch AM, Roobottom CA, Morgan-Hughes GJ. Imaging the heart valves using ECG-gated 64detector row cardiac CT. Br J Radiol. (2008) 81:275-90. doi: 10.1259/bjr/ 16301537

37. Kang SH, Kim MK, Kim HJ, Zhengguo P, Lee SH. Accuracy assessment of image-based surface meshing for volumetric computed tomography images in the craniofacial region. J Craniofac Surg. (2014) 25:20515. doi: $10.1097 /$ SCS.0000000000001139

38. Ye N, Long H, Zhu S, Yang Y, Lai W, Hu J. The accuracy of computer imageguided template for mandibular angle ostectomy. Aesthetic Plast Surg. (2015) 39:117-23. doi: 10.1007/s00266-014-0424-1

39. Sun Y, Luebbers HT, Agbaje JO, Schepers S, Vrielinck L, Lambrichts I, et al. Accuracy of upper jaw positioning with intermediate splint fabrication after virtual planning in bimaxillary orthognathic surgery. J Craniofac Surg. (2013) 24:1871-6. doi: 10.1097/SCS.0b013e31829a80d9 
40. Ono I, Abe K, Shiotani S, Hirayama Y. Producing a full-scale model from computed tomographic data with the rapid prototyping technique using the binder jet method: a comparison with the laser lithography method using a dry skull. J Craniofac Surg. (2000) 11:527-37. doi: 10.1097/00001665-200011060-00004

41. Otawa N, Sumida T, Kitagaki H, Sasaki K, Fujibayashi S, Takemoto M, et al. Custom-made titanium devices as membranes for bone augmentation in implant treatment: Modeling accuracy of titanium products constructed with selective laser melting. J Craniomaxillofac Surg. (2015) 43:128995. doi: 10.1016/j.jcms.2015.05.006

42. Hazeveld A, Huddleston Slater JJ, Ren Y. Accuracy and reproducibility of dental replica models reconstructed by different rapid prototyping techniques. Am J Orthod Dentofacial Orthop. (2014) 145:108-15. doi: 10.1016/j.ajodo.2013.05.011

43. Chen G, Jiang M, Coles-Black J, Mansour K, Chuen J, Amott D. Threedimensional printing as a tool in otolaryngology training: a systematic review. J Laryngol Otol. (2020) 134:14-9. doi: 10.1017/S0022215119002585

44. Gallagher AG, Ritter EM, Satava RM. Fundamental principles of validation, and reliability: rigorous science for the assessment of surgical education and training. Surg Endosc. (2003) 17:1525-9. doi: 10.1007/s00464-003 0035-4

45. Kemp S, Coles-Black J, Walker MJ, Wallace G, Chuen J, Mukherjee P. Ethical and regulatory considerations for surgeons as consumers and creators of three-dimensional printed medical devices. ANZ J Surg. (2020) 90:1477-81. doi: 10.1111/ans. 15871

Conflict of Interest: The authors declare that the research was conducted in the absence of any commercial or financial relationships that could be construed as a potential conflict of interest.

Copyright (c) 2021 Coles-Black, Bolton and Chuen. This is an open-access article distributed under the terms of the Creative Commons Attribution License (CC BY). The use, distribution or reproduction in other forums is permitted, provided the original author(s) and the copyright owner(s) are credited and that the original publication in this journal is cited, in accordance with accepted academic practice. No use, distribution or reproduction is permitted which does not comply with these terms. 